\title{
Aberrant expression of class III B-tubulin in basal cell carcinoma of the skin
}

\author{
MITSUAKI ISHIDA $^{1}$, RYOJI KUSHIMA ${ }^{2}$ and HIDETOSHI OKABE ${ }^{1,2}$ \\ ${ }^{1}$ Department of Clinical Laboratory Medicine and ${ }^{2}$ Division of Diagnostic Pathology, \\ Shiga University of Medical Science, Shiga, Japan
}

Received April 9, 2009; Accepted June 5, 2009

DOI: 10.3892/or_00000494

\begin{abstract}
Tubulin is a major component of microtubules. Class III $\beta$-tubulin ( $ß$ III) is a neuron-associated $\beta$-tubulin isotype and expressed in the normal central and peripheral nervous systems. According to a previous study, B III is not expressed in normal skin and squamous cell carcinoma. However, its expression has not been examined in basal cell carcinoma (BCC) of the skin. Expression of B III was analyzed together with neural cell adhesion molecule (NCAM), chromogranin A, synaptophysin, epithelial membrane antigen (EMA) and cytokeratin (CK) 20 by immunohistochemical methods in 10 non-neoplastic skin tissues and 50 BCCs. In the normal skin, immunoreactivity to $\beta$ III was restricted to the nerve bundles in the dermis and subcutis, no positivity was shown in epithelial cells of the epidermis and skin appendages. $\beta$ III and NCAM were expressed in 50 and $68 \%$ of BCCs, respectively, predominantly periphery of tumor nests, although the distribution of both markers was not always identical. Chromogranin A, synaptophysin and CK 20 were not expressed in any of BCCs. EMA was focally expressed in only $8 \%$ of BCCs. B III is a potential candidate for inclusion to the panel of immunohistochemical markers to distinguish small BCCs from non-neoplastic hair buds, because non-neoplastic hair follicles are not positive for $B$ III.
\end{abstract}

\section{Introduction}

Basal cell carcinoma (BCC) of the skin, one of the most common cutaneous carcinoma, has been proposed to originate from follicular germinative cells according to the expression of various cytokeratins and other markers (1-5). Previous immunohistochemical analyses suggested that the minority of BCCs showed neuroendocrine differentiation $(6,7)$. Although

Correspondence to: Dr Hidetoshi Okabe, Department of Clinical Laboratory Medicine and Division of Diagnostic Pathology, Shiga University of Medical Science, Seta, Tsukinowa-cho, Otsu, Shiga 520-2192, Japan

E-mail: okabe@belle.shiga-med.ac.jp

Key words: basal cell carcinoma, class III ß-tubulin, skin neuroendocrine cells and neural tissues frequently share common immunohistochemical markers, the neuroendocrine markers have not yet been completely examined in BCCs.

Tubulin is a major component of microtubules, which are ubiquitous cytoskeltal structures deeply involved in the intracellular transport, cell movement and mitosis. Tubulin consists of $\alpha$ and $\beta$ subunits. In mammals, six $\beta$-tubulin isotypes have been identified and the complex variable patterns of their expression denote cellular and functional specificity and diversity (8).

Class III $\beta$-tubulin ( $ß$ III) is a neuron-associated isotype that is considered to be one of the earliest neuron-associated cytoskeletal markers and is thought to play an important functional role in neuronal morphogenesis (8). ß III expression is observed throughout the lifetime from early development in the central and peripheral nervous systems and in some nerve tumors, such as high-grade gliomas (9), oligodendrogliomas (10), medulloblastomas (11), retinoblastomas (12), and pheochromocytomas (13). B III expression was also shown in the non-neuronal tissues and their tumors, for example: fetal Kulchitsky cells, which are neuroendocrine cells of the lung; neuroendocrine cell tumors of the lung, such as small cell carcinomas, large cell neuroendocrine cell carcinomas and some of atypical carcinoid tumors (14); certain gastrointestinal carcinoid tumors (15); and various lung carcinomas, especially adenocarcinomas (14). Although $\beta$ III expression was not revealed in the normal skin, its appendages and squamous cell carcinoma of the skin (16), its expression in $\mathrm{BCC}$ has not yet been examined. Here, we report the expression of $\mathrm{B}$ III in BCCs, in comparison to that of neural cell adhesion molecule (NCAM), chromogranin A, synaptophysin, epithelial membrane antigen (EMA) and cytokeratin (CK) 20, and discuss the diagnostic utility of examination of ß III expression.

\section{Materials and methods}

Case selection. The BCC cases in this study comprised of 50 consecutive operative specimens from 49 patients from Shiga University of Medical Science Hospital obtained during 20042008. These cases were diagnosed by at least two diagnostic pathologists approved by the Japanese Society of Pathology. The median age of the patients (29 men and 20 women) was 73.2 years (range 15-95 years). One patient had 2 different lesions on the face and a 15-year-old patient had a previous 



Figure 1. Immunohistochemistry of class III B-tubulin in non-neoplastic skin. (A) Positive immunoreactivity is confined to the nerve bundles in the subcutis. (B) Hair follicles, (C) sweat glands and (D) sebaceous glands are negative for B III, but nerve bundles (arrow) are positive (C). Original magnifications, (A) $\mathrm{x} 400$, (B) $\mathrm{x} 200$, (C) $\mathrm{x} 200$ and (D) $\mathrm{x} 400$.
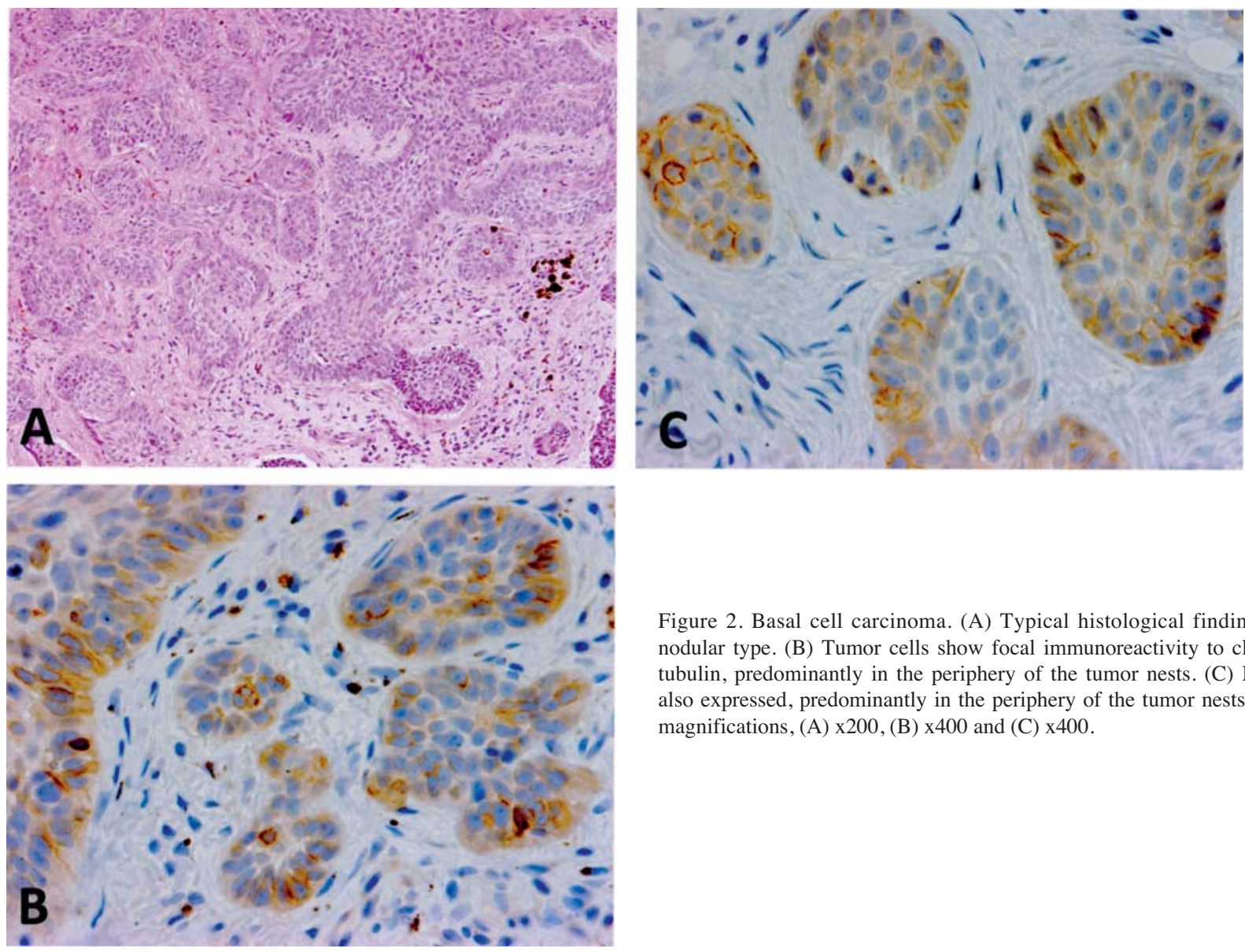

Figure 2. Basal cell carcinoma. (A) Typical histological findings of the nodular type. (B) Tumor cells show focal immunoreactivity to class III Btubulin, predominantly in the periphery of the tumor nests. (C) NCAM is also expressed, predominantly in the periphery of the tumor nests. Original magnifications, (A) x200, (B) x400 and (C) x400. 
Table I. Immunohistochemical results of basal cell carcinomas.

\begin{tabular}{|c|c|c|c|c|c|c|}
\hline & \multicolumn{3}{|c|}{ Class III ß-tubulin } & \multicolumn{3}{|c|}{ NCAM } \\
\hline & - & $1+$ & $2+$ & - & $1+$ & $2+$ \\
\hline Nodular & $14 / 31$ & $17 / 31$ & $0 / 31$ & $9 / 31$ & $17 / 31$ & $5 / 31$ \\
\hline Micronodular & $4 / 7$ & $3 / 7$ & $0 / 7$ & $2 / 7$ & $5 / 7$ & $0 / 7$ \\
\hline Superficial & $6 / 9$ & $3 / 9$ & $0 / 9$ & $4 / 9$ & $5 / 9$ & $0 / 9$ \\
\hline Infiltrative & $1 / 3$ & $2 / 3$ & $0 / 3$ & $1 / 3$ & $2 / 3$ & $0 / 3$ \\
\hline
\end{tabular}

diagnosis of xeroderma pigmentosum. The examined specimens were derived from the face (40 cases), trunk ( 6 cases), scalp ( 3 cases) and thigh ( 1 case).

The diagnostic criteria for BCC were based on the description in the World Health Organization Classification of Tumors, Pathology and Genetics of Skin Tumors (17) as follows: lobules, columns, bands and cords of basaloid cells (germinative cells) associated with scant cytoplasm and a characteristic outer palisade of cells associated with a surrounding loose fibromucinous stroma. In addition, artefactual retraction spaces between the tumor and stroma are present, and apoptosis is usually observed. These cases were subclassified by the World Health Organization Classification of Tumors, Pathology and Genetics of Skin Tumors (17) as superficial, nodular, micronodular or infiltrative types.

Ten non-neoplastic skin tissue samples (5 from the scalp and 5 from the face) from perilesional excision areas were also used. None of these tissues displayed any pathological findings.

Immunohistochemistry. Immunostaining was performed using an autostainer (XT System Benchmark, Ventana Medical System, Tucson, AZ, USA) according to the manufacturer's instruction. Primary antibodies used in the study were a mouse monoclonal antibody against human chromogranin A (diluted 1:200; DAK-A3, Dako Cytomation, Glostrup, Denmark), a mouse monoclonal antibody against human class III B-tubulin (diluted 1:400; TU-20, Chemicon International Inc., Temecula, CA, USA), a mouse monoclonal antibody against human neural cell adhesion molecule (diluted 1:100; CD564, Novocastra Laboratories Ltd., Newcastle upon Tyne, UK), a mouse monoclonal antibody against human synaptophysin (diluted 1:400; 27G12, Novocastra), a mouse monoclonal antibody against human epithelial membrane antigen (diluted 1:300; GP1.4, Novocastra), and a mouse monoclonal antibody against human cytokeratin 20 (dilated 1:50; Ks20.8, Novocastra). Sections for the all antibodies were pretreated with heat.

Immunohistochemical findings were verified by control studies. Dorsal root ganglia were used as the outer positive control for B III and NCAM, and nerve bundles around the tumor tissue were also used as the inner positive control for both markers. Appendiceal carcinoid tumors were used as the outer positive control for chromogranin A and synaptophysin. Squamous cell carcinomas of the skin were used as the outer positive control for EMA, and sebaceous and eccrine glands around the tumor tissue were also used as the inner positive control for EMA. Colorectal carcinomas were used as the outer positive control for CK 20. Negative controls were evaluated by substituting the primary antibody with similar diluted non-immunized mouse serum. Immunohistochemical staining of $B$ III and other markers was carried out in serial sections to evaluate the correlation between marker distributions.

Evaluation of immunoreactivity. The degree of immunoreactivity to each monoclonal antibody was classified into the following three categories: 0 (no positive cells in the tumor),+1 (positive cells comprised of less than $50 \%$ of the tumor), and +2 (positive cells comprised of more than $50 \%$ of the tumor).

\section{Results}

Normal skin. Positive immunoreactivity to $ß$ III was confined to the nerve bundles in the dermis and subcutis (Fig. 1A). None of the epithelial cells of the epidermis, hair follicles (Fig. 1B), including matrical cells, outer root sheath, and inner root sheath, sweat glands (Fig. 1C) and sebaceous glands (Fig. 1D) were positive for $\beta$ III.

NCAM was expressed only in the nerve bundles in the dermis and subcutis, but not expressed in the epithelial components except in Merkel cells of the epidermis and hair follicles. Chromogranin A, synaptophysin and CK 20 were not expressed in the epithelial cells of the epidermis and skin appendages except in Merkel cells. EMA was expressed in the sebaceous and eccrine glands, but was not expressed in the epithelial cells of the epidermis and hair follicles.

BCCs. Subclassification of BCCs is as follows: 28 cases from the face, 2 cases from the scalp and 1 case from the thigh were of the nodular type (Fig. 2A); 7 cases from the face were of the micronodular type; 6 cases from the trunk and 3 cases from the face were of the superficial type; and 2 cases from the face and 1 case from the scalp were of the infiltrative type.

Table I summarizes the immunohistochemical results for $\beta$ III and NCAM. B III was focally expressed in 25 of 50 cases $(50 \%)$. The immunoreactivity of $\beta$ III was predominantly distributed in the periphery of the tumor nests (Fig. 2B). Further, 55\% of nodular type (17/31 cases), $43 \%$ of micronodular type (3/7 cases), $33 \%$ of superficial type (3/9 cases) and $67 \%$ of infiltrative type (2/3 cases) showed positive immunoreactivity to $\beta$ III (Table I). There was no significant correlation between the histological subtypes and the incidence of $B$ III expression (Table I). 
Table II. The correlation of class III B-tubulin and NCAM immunoreactivity.

Class III ß-tubulin

\begin{tabular}{cccc} 
& & Positive & Negative \\
\hline \multirow{2}{*}{ NCAM } & Positive & 18 & 16 \\
& Negative & 7 & 9 \\
\hline
\end{tabular}

NCAM was expressed in 34 of 50 cases $(68 \%)$ and its expression was found in all four subtypes (Table I). Most cases showed focal immunoreactivity to NCAM, predominantly in the periphery of the tumor nests, but 5 cases of nodular type had diffuse immunoreactivity (Table I). Table II summarizes the correlation of B III and NCAM immunoreactivity. In 34 NCAM-positive cases (including +1 and +2), 18 cases were both NCAM and $\beta$ III positive, while 16 cases were $\beta$ III negative (Table II). In 25 B III-positive cases, 7 cases were NCAM negative (Table II). In the cases which were both $B$ III and NCAM positive, the distribution of these two markers was not always identical.

Chromogranin A, synaptophysin and CK 20 were not expressed in all of the $50 \mathrm{BCC}$ cases in our series, and no CK 20-positive Merkel cells were found in the 50 BCCs. EMA was focally expressed (+1) in only 4 cases of our series ( 3 cases of nodular type and 1 case of micronodular type).

\section{Discussion}

Class II $\beta$-tubulin ( $ß$ II), a molecule that belongs to the tubulin superfamily, is a neural type tubulin isotype and is also known as the 'major brain tubulin' (8). It is widely distributed in the developing neuronal axons and dendrites as well as neuroepithelial tumors and represents a marker for progenitor and neuronal stem cells (18). Roh et al reported that $\beta$ II was focally expressed in approximately half of BCCs (32/57 cases) (19). However, B II expression is not directly associated with neuroendocrine differentiation in the cutaneous tumors, because B II is expressed in the keratinocytes of the granular layer, hair cortical and cuticular cells, inner root sheath and companion layer of the outer root sheath in the normal skin and up-regulated during squamous differentiation of keratinocytes (20). Roh et al also reported that $B$ II expression was increased in the areas of squamous or follicular differentiation in the cutaneous tumors (19). Thus, B II expression in BCCs is likely not the predominant phenotype related to the neuroendocrine differentiation.

B III, a $50 \mathrm{kDa}$ protein, also belongs to the tubulin superfamily and is generally referred to as the 'minor brain tubulin', as compared to $B$ II $(8,16)$. It has been used as a marker of early phase of neuronal differentiation in developmental and pathological studies. Unlike $\beta$ II, a previous report showed that $\beta$ III was not expressed in the normal epithelial cells of the epidermis, hair follicles, sweat glands and sebaceous glands (16), which are identical to the results of our present study. On the other hand, we demonstrated aberrant expression of $B$ III in $50 \%$ of BCC cases and there was no significant correlation between the histological subtypes of BCCs and the incidence of $\beta$ III expression. This is the first report to show $B$ III expression in the cutaneous tumor.

B III is expressed in certain neuroendocrine tumors, especially high grade neuroendocrine tumors of the lung (14), gastrointestinal carcinoid tumors (15), and nonneuroendocrine carcinomas of the lung, in particular adenocarcinomas (14). Katsetos et al hypothesized that the aberrant expression of $\beta$ III in non-neuronal tumors may highlight alteration in the isotype composition of $\beta$-tubulin in the tumor subclones (8). This may dictate or predict the direction of tumor behavior and chemoresponsiveness to microtubuleacting compounds (8). Cloning and characterization of the promoter region of the rat $\beta$ III have been performed, which will likely provide important insights into potential mechanism of the $B$ III expressional regulation (21).

Microtubule associated proteins (MAPs), a group of cytoskeletal protein, play an important role in assembly of the microtubules (22). While the tubulin isoforms form the frame of the network, the integrity and intracellular organization of the microtubule network are modulated and assembled by MAPs. MAP-2 is a member of the MAP families and is expressed mainly in neurons (22). MAP-2 has repetitive microtubule-binding domains, which are believed to help MAP-2 protein confer stability to the microtubule (22). MAP-2 expression is very weak in neuronal precursors, but becomes stronger about 1 day after expression of $B$ III since MAP-2 functions to stabilize $B$ III (23). A recent study revealed that MAP-2 expression was observed in the hair follicle, almost exclusively in the innermost layer of the outer root sheath of the anagen follicle, and in the upper layers of the nail matrix in the normal skin (24). In addition, Liu et al demonstrated that MAP-2 was expressed in 100\% of nevi, $87 \%$ of malignant melanomas, $60 \%$ of desmoplastic melanomas (25) and also in all Merkel cell carcinomas (26). They also reported that no positive immunoreactivity to MAP-2 was found in any BCCs (0/20 cases) (26). The expression of MAP-2 in non-neoplastic skin and BCCs $(24,26)$ is different from that of $ß$ III. Accordingly, further analyses are needed to clarify the molecular mechanism of the aberrant expression of $B$ III in BCC. However, our results suggest that the examination of the aberrant expression of $B$ III may be useful for distinguishing BCC from non-neoplastic hair buds, because non-neoplastic hair follicles are not positive for B III.

NCAM expression was observed in $68 \%$ of our 50 BCCs, and this result corroborates the previous report by Chen-Tsai et al (27). Distribution of $\beta$ III and NCAM was not always identical (Table II). In addition, a few reports previously demonstrated that the minority of BCC cases showed neuroendocrine differentiation $(6,7)$. George et al reported that chromogranin A was expressed in only 4\% (2/53 cases) of BCCs (6). However, chromogranin A and synaptophysin were not expressed in any of our $50 \mathrm{BCCs}$, including the $B$ III and/or NCAM-positive cases. $ß$ III expression is observed in various neuroendocrine tumors of the lung and gastrointestinal tract $(14,15)$, but our results show that $B$ III expression in $\mathrm{BCC}$ does not have high correlation with neuroendocrine differentiation. In addition, EMA was focally expressed in 
only $8 \%$ (4/50 cases) of BCCs. It is well known that the expression of EMA is uncommon in BCCs $(28,29)$, which corroborates the results of our present study.

Schulz and Hartshuh reported that CK 20, a marker for Merkel cells, was useful for differentiating BCCs from trichoblastomas, because Merkel cells are rare or absent in the BCCs, in contrast to trichoblastomas which have scattered CK 20-positive Merkel cells (29). In our series, none of the 50 BCCs had CK20 positive-Merkel cells, and CK20positive tumor cells were not observed. This result is relevant to a previous report (30).

In conclusion, we demonstrated that aberrant expression of $\beta$ III was observed in $50 \%$ of BCCs, although $\beta$ III was not expressed in the normal epithelial cells of the epidermis, hair follicles, sweat glands and sebaceous glands. Our results suggest the examination of the aberrant expression of $B$ III may be useful for distinguishing BCC from non-neoplastic hair buds. Further studies are needed to clarify the molecular mechanism of the aberrant expression of $\beta$ III in BCC.

\section{References}

1. Asada M, Schaart FM, de Almeida HJ Jr, et al: Solid basal cell epithelioma (BCE) possibly originates from the outer root sheath of the hair follicle. Acta Derm Venereol 73: 286-292, 1993.

2. Kurzen H, Esposito L, Langbein L and Hartschuh W: Cytokeratins as markers of follicular differentiation: an immunohistochemical study of trichoblastoma and basal cell carcinoma. Am J Dermatopathol 23: 501-509, 2001.

3. Krüger K, Blume-Peytavi U and Orfanos CE: Basal cell carcinoma possibly originates from the outer root sheath and/or the bulge region of the vellus hair follicle. Arch Dermatol Res 291: 253-259, 1999.

4. Jih DM, Lyle S, Elenitsas R, Elder DE and Cotsarelis G: Cytokeratin 15 expression in trichoepitheliomas and s subset of basal cell carcinomas suggests they originate from hair follicle stem cells. J Cutan Pathol 26: 113-118, 1999.

5. Ishida M, Kushima R and Okabe H: Immunohistochemical demonstration of D2-40 in basal cell carcinomas of the skin. J Cutan Pathol 35: 926-930, 2008.

6. George E, Swanson PE and Wick MR: Neuroendocrine differentiation in basal cell carcinoma. An immunohistochemical study. Am J Dermatopathol 11: 131-135, 1989.

7. Foschini MP and Eusebi V: Divergent differentiation in endocrine and nonendocrine tumor of the skin. Semin Diagn Pathol 17: 162-168, 2000.

8. Katsetos CD, Herman MM and Mörk SV: Class III ß-tubulin in human development and cancer. Cell Motil Cytoskeleton 55: 77-96, 2003.

9. Katsetos CD, Del Valle L, Geddes JF, et al: Aberrant localization of the neuronal class III $\beta$-tubulin in astrocytomas: a marker for anaplastic potential. Arch Pathol Lab Med 125: 613-624, 2001

10. Katsetos CD, Del Valle L, Geddes JF, et al: Localization of the neuronal class III B-tubulin in oligodendrogliomas: comparison with Ki-67 proliferative index and 1p/19q status. J Neuropathol Exp Neurol 61: 307-320, 2002.

11. Katsetos CD, Krishna L, Frankfurter A, et al: A cytomorphological scheme of differentiating neuronal phenotypes in cerebellar medulloblastomas based on immunolocalization of class III $\beta$-tubulin isotype ( $\beta$ III) and proliferating cell nuclear antigen (PCNA)/cyclin. Clin Neuropathol 14: 72-81, 1995.
12. Katsetos CD, Herman MM, Frankfurter A, Uffer S, Perentes E and Rubinstein LJ: Neuron-associated class III B-tubulin isotype, microtubule-associated protein 2 , and synaptophysin in human retinoblastomas in situ. Further immunohistochemical observations on the Flexner-Wintersteiner rosettes. Lab Invest 64: 45-54, 1991.

13. Karkavelas G, Katsetos CD, Geddes JF, et al: Class III ß-tubulin isotype ( $(\mathrm{III})$ in the adrenal medulla: II. Localization in primary human pheochromocytomas. Anat Rec 250: 344-350, 1998.

14. Katsetos CD, Kontogeorgos G, Geddes JF, et al: Differential distribution of the neuron-associated class III B-III tubulin in neuroendocrine lung tumors. Arch Pathol Lab Med 124: 535-544, 2000.

15. Jirasek T, Mandys V and Viklicky V: Expression of class III ßtubulin in neuroendocrine tumors of gastrointestinal tract. Folia Histochem Cytobiol 40: 305-310, 2002.

16. Draberova E, Lukas Z, Ivanyi D, Viklicky V and Draber P: Expression of class III $\beta$-tubulin in normal and neoplastic human tissues. Histochem Cell Biol 109: 231-239, 1998.

17. Kossard S, Epstein EH Jr, Cerio R, Yu LL and Weedon D: Basal cell carcinoma. In: World Health Organization Classification of Tumors, Pathology and Genetics of Skin Tumors. LeBoit PE, Burg G, Weedon D and Sarasin A (eds). IARC Press, Lyon, pp13-19, 2006.

18. Sugita Y, Nakamura Y, Yamamoto M, Oda E, Tokunaga O and Shigemori M: Expression of tubulin B II in neuroepithelial tumors: reflection of architectural changes in the developing human brain. Acta Neuropathol 110: 127-134, 2005.

19. Roh JY, Kee SH, Choi JW, et al: Expression of class II Btubulin in non-melanoma cutaneous tumors. J Cutan Pathol 34: $166-173,2007$

20. Lee WH, Kim JY, Kim YS, et al: Upregulation of class II Btubulin expression in differentiating keratinocytes. J Invest Dermatol 124: 291-297, 2005.

21. Dennis K, Uittenbogaard M, Chiaramello A and Moody SA: Cloning and characterization of the 5'-flanking region of the rat neuron-specific Class III B-tubulin gene. Gene 294: 269-277, 2002.

22. Maccioni RB and Cambiazo V: Role of microtubule-associated proteins in the control of microtubule assembly. Physiol Rev 75: 835-864, 1995.

23. Dehmelt L and Halpain S: The MAP2/Tau family of microtubule-associated proteins. Genome Biol 6: 204-213, 2004.

24. Hallman JR, Fang D, Setaluri V and White WL: Microtubule associated protein (MAP-2) expression defines the companion layer of the anagen hair follicle and an analogous zone in the nail unit. J Cutan Pathol 29: 549-556, 2002.

25. Liu Y, Saad RS, Shen SS and Silverman JF: Diagnostic value of microtubule-associated protein-2 (MAP-2) for neuroendocrine neoplasms. Adv Anat Pathol 10: 101-106, 2003.

26. Liu Y, Mangini J, Saad R, et al: Diagnostic value of microtubule-associated protein-2 in Merkel cell carcinoma. Appl Immunohistochem Mol Morphol 11: 326-329, 2003.

27. Chen-Tsai CP, Colome-Grimmer M and Wagner RF Jr: Correlations among neural cell adhesion molecule, nerve growth factor, and its receptors, TrkA, TrkB, TrkC, and p75NGFR, in perineural invasion by basal cell and cutaneous squamous cell carcinomas. Dermatol Surg 30: 1009-1016, 2004.

28. Fan YS, Carr RA, Sanders DSA, Smith AP, Lazar AJF and Calonje E: Characteristic Ber-EP4 and EMA expression in sebaceoma is immunohistochemically distinct from basal cell carcinoma. Histopathology 51: 80-86, 2007.

29. Beer TW, Shepherd P and Theaker JM: Ber EP4 and epithelial membrane antigen aid distinction of basal cell, squamous cell and basosquamous carcinomas of the skin. Histopathology 37 : 218-223, 2000

30. Schulz T and Hartschuh W: Merkel cells are absent in basal cell carcinomas but frequently found in trichoblastomas. An immunohistochemical study. J Cutan Pathol 24: 14-24, 1997. 